\title{
Modeling the risk of heat illness among basic training populations within the DoD, 2010-2017
}

\author{
Jessica F. Deerin*, Vivek Khatri and Paul E. Lewis
}

Armed Forces Health Surveillance Branch, Alexandria, VA, USA

\section{Objective}

To identify predictors of the risk of developing exertional heat illness (EHI) among basic training populations in the Department of Defense.

\section{Introduction}

Although effective preventive measures for heat-related illness have been recommended and mandated for military personnel, there continues to be incident cases. In 2016, there were 401 incident cases of heat stroke and 2,135 incident cases of "other heat illness" among all active component service members. Current military guidelines utilize the wet bulb globe temperature (WBGT) index to measure heat risk, guiding work/rest and hydration practices. The WBGT requires calibrated instrumentation and is based on fixed cutoff values. We propose using readily available meteorological data inputs and EHI cases to identify and validate an EHI risk prediction model. Prior studies have found that combinations of WBGT and the previous day's WBGT and relative humidity and temperature have predictive value for EHI. ${ }^{1}$ We build upon prior work by using generalized additive models (GAMs).

\section{Methods}

A case-control study was conducted among active component service members from all basic training installations from January 1, 2010 to May 31, 2017. Incident cases of EHI were identified utilizing diagnosis codes extracted from inpatient and outpatient medical encounters and confirmed reportable medical events. An equal number of random controls, matched by installation, were selected. Mean weather data during daylight hours from the Air Force Weather Squadron were provided for the closest weather station to the installation during the same time period. A GAM was used due to the non-linear association between EHI and weather predictors, to develop models for the risk of incident EHI. Training (75\% of data) and test ( $25 \%$ of data) datasets were generated for model training and model validation. Three hundred sets of training and test datasets were randomly generated. For each set, sensitivity and specificity for EHI prediction was calculated. Four models with different combinations of predictors were compared: model 1 contains month, day of week, and installation; model 2 contains WBGT, month, day of week, and installation; model 3 contains WBGT, previous day's WBGT, month, day of week, and installation; and model 4 contains relative humidity, temperature, month, day of week, and installation. Each predictor was significantly associated with EHI. The mean differences in sensitivity and specificity between all models and model 1 were compared and $95 \%$ confidence intervals were generated by bootstrapping. GAMs were generated using the $\mathrm{mgcv}$ package and odds ratios were generated using the oddsratio package in $\mathrm{R}$.

\section{Results}

There were 5,258 incident cases of EHI from 2010-2017 among active component service members stationed at basic training installations. There was not a significant difference in model performance when comparing the four models. The mean differences in sensitivity and specificity of each model compared to model 1 are displayed in Table 1. The association between log odds of EHI and
WBGT, controlling for month, day of week, and installation (model 2 ) is displayed in Figure 1. There is not a single representative odds ratio generated for GAMs due to the non-linear relationship between predictors and the log odds of EHI. As an example, the odds ratio between two arbitrary WBGT points is displayed. The odds of EHI among those exposed to a mean WBGT of $85^{\circ} \mathrm{F}$ is $2.55(95 \% \mathrm{CI}$ : $2.45,2.64)$ times the odds of EHI among those exposed to a mean WBGT of $80^{\circ} \mathrm{F}$. The association between the log odds of EHI and relative humidity, controlling for month, day of week, installation, and temperature (model 4 ) is displayed in Figure 2. The odds of EHI among those exposed to $80 \%$ relative humidity is 1.36 (95\% CI: 1.33 , 1.39) times the odds of EHI among those exposed to $60 \%$ relative humidity.

\section{Conclusions}

Our results provide evidence that there is no significant difference in model prediction of EHI utilizing various combinations of weather predictors. However, there is a significant non-linear association between weather predictors and EHI and examples of these relationships are given using different models. Model performance can be improved by including more granular exposure data (i.e. physical activity during EHI episode, biometric and physiological measures).

Table 1. Generalized additive model comparison: mean sensitivity and specificity

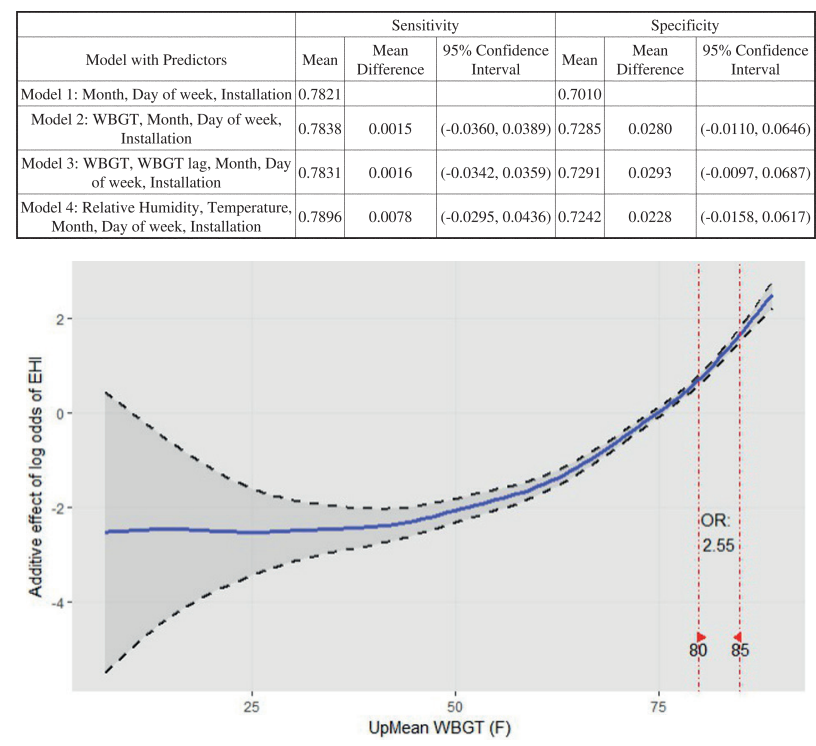

Figure 1. Association between log odds of EHI and WBGT, controlling for month, day of week, and installation 


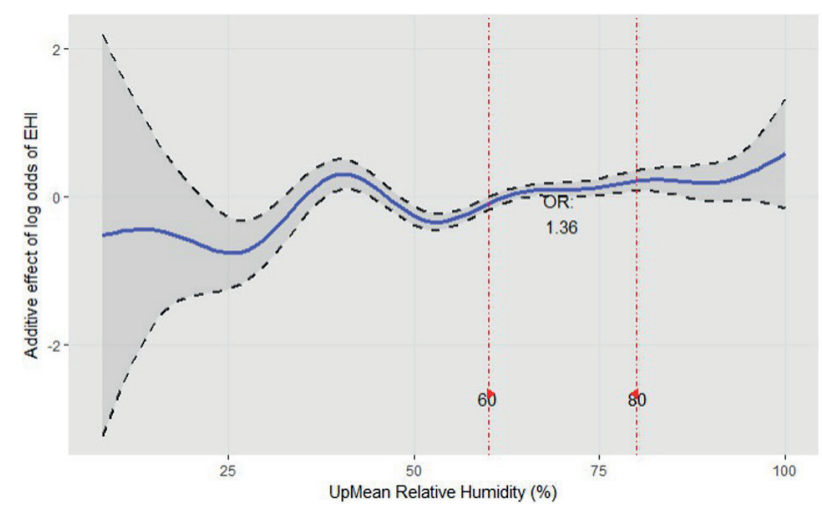

Figure 2. Association between log odds of EHI and mean relative humidity, controlling for month, day of week, installation, and temperature

\section{Keywords}

prediction; heat illness; weather; military

\section{References}

1. Wallace RF, Kriebel D, Punnett L, Wegman DH, Wenger CB, Gardner JW, Gonzalez RR. The effects of continuous hot weather training on risk of exertional heat illness. Med Sci Sports Exerc. 2005 Jan; 37(1):84-90.

\section{*Jessica F. Deerin}

E-mail: jessica.deerin@gmail.com 